\title{
Parenting Across Diverse Contexts
}

\author{
Alfiee M. Breland-Noble
}

Published online: 29 December 2013

(c) Springer Science+Business Media New York 2013

\begin{abstract}
This article serves as an introduction to a special issue focused on parenting in diverse cultural groups worldwide. Within this editorial, I describe popular culture presentations of parenting practices and discuss these in the context of applicability and generalizability for diverse racial, cultural and socioeconomic populations. I present background on some of the seminal research in parenting, describe the impetus for the special issue and provide a brief overview of the manuscripts selected for inclusion in this special issue of the Journal of Child and Family Studies.
\end{abstract}

Keywords Parenting · Parent training · Parent-child relationships · Child and adolescent mental health

\section{Introduction}

The impetus for this Special Issue came from discussions I shared with the Editor-in-Chief as I was preparing a Special Issue on African American youth mental health. As I vetted and processed manuscripts for that Special Issue, I noted a number of submissions focused on parenting in African American families. Concurrently, in my role as Associate Editor for the journal, I noticed a similar trend of research submissions related to parenting in other manuscripts assigned to me. The theme that held these manuscripts together, even within a journal focused on parents, youth and families, was their specific attention to illuminating the

A. M. Breland-Noble ( $\square)$

Department of Psychiatry, Georgetown University Medical

Center, 2115 Wisconsin Avenue Suite 120, Washington, DC 20007, USA

e-mail: ab2892@georgetown.edu concerns that parents, guardians, fictive kin and others face as they engage in one of the most important, challenging and rewarding tasks in life: the task of child rearing. As I reflected on how the journal could support the literature and the general public in discussing issues relevant for parents and families, I considered the challenges that parents face. Interestingly, though we live in an increasingly global society filled with technological advances and ever improving mechanisms for gathering new information, no parent arrives at home with a newborn and a detailed instruction manual for how to ensure the health, welfare and safety of that new and precious gift. Instead, we are tasked with mustering all of our personal, familial, cultural and societal resources to do the best we can in showing love and affection and ensuring the safety and well being of a new little person.

For parents of racially, culturally and socioeconomically diverse backgrounds, there are the added concerns related to ensuring that their children (who are not of the majority culture, or who may lack social capital and resources) are equipped to function and excel in a society where they may be marginalized or experience discrimination.

Arguably, the seminal work on parenting styles is Diana Baumrind's typology. Baumrind's research reminds us that parents have varying approaches to child rearing focused on how parents teach their children and express their affection. She initially named three parenting styles: permissive, authoritarian and authoritative and, many years later, Maccoby and Martin expanded her typology to include Indulgent and Neglectful. Overall, a strong body of research indicates that these varying parenting styles impact children's emotional development and overall mental health throughout childhood and into adulthood (Maccoby and Martin 1983).

While Baumrind, Maccoby and Martin base their assertions about parenting on observations conducted using 
the scientific method, popular culture often distills research findings into sensational sound bites. For example, in recent years there has been significant controversy surrounding ideas like "tiger parenting", spanking and "harsh verbal punishment". Interestingly, while media reports on such "hot button" parenting topics catch the public's attention, rarely do the discussions involve an in-depth, well-contextualized and deep examination of the topics at hand. All of this can make sifting through the advice quite difficult for any parent and contributes to parental worry about their behaviors. In one way, my hope is that this Special Issue can assist us in bridging this gap between quick news sound bites and commentary on parenting that may not come from objective observations. For example, in 2011 Amy Chua published "Battle Hymn of the Tiger Mother" about her experiences with (what has come to be called) "tiger parenting" or a parenting approach that involves setting extremely high expectations for children, maintaining very strict discipline and remaining emotionally unsupportive or distant from one's children. In response, a number of researchers (mostly Asian and Asian American) published studies challenging the approach to parenting as well as its assumed prevalence among Asian and Asian American parents. Their research dispels stereotypes about the prevalence of this authoritarian approach to parenting and indicates quite clearly that it has far more negative outcomes for youth mental health and well-being than positive (Juang et al. 2013; Kim et al. 2013).

A second recent research study (as it was reported in the mainstream media) argued that children who were yelled at by their parents experienced poor mental health outcomes comparable to those of peers who were physically punished by their parents. Again, on the surface, the story draws a direct link between yelling and poor adolescent outcomes. However, when one reviews the study in depth, one finds that a group of factors together are correlated with worse outcomes for youth. In other words, one must read the primary study to understand exactly how the authors define "harsh verbal punishment" (i.e., name-calling, indiscriminant screaming or cursing) and other behaviors that are consistently associated with abuse. Unfortunately, this type of in-depth analysis of findings is rarely presented to parents in the lay public in a manner that clearly delineates the concepts of import (in this case harsh verbal punishment) in a way that gives parents clear and useful take home messages. Additionally, for parents of racially and socioeconomically diverse backgrounds, rarely does research attempt to create a balanced picture of their unique parenting experiences and the impact of those experiences on their children. Over 40 years ago, Baumrind herself cautioned against generalizing findings from parenting research with majority culture persons to non-majority populations in the absence of considering psychosocial factors (like race, culture and socioeconomic status) known to impact parents, youth and families. This is sage advice that we attempt to utilize in the current Special Issue.

\section{Contents of the Special Issue}

I have developed this Special Issue of the Journal of Child and Family Studies as a means of synthesizing a body of work related to parenting from a global perspective. While parents and caregivers face a multitude of issues in meeting the milestones associated with rearing children, they must consider just as many options for effectively meeting the task. In response, we present a range of perspectives on parenting by examining five aspects including: (1) psychosocial factors impacting parents and families; (2) comparisons of parenting styles and quality/type of parental interpersonal relationships; (3) parent-child interpersonal relationships; (4) the impact of physical and mental illness on parents and youth; and (5) parenting interventions.

In keeping with a focus on interpersonal relationships between parents and youth, Pearl, French, Dumas, Moreland and Prinz describe the bidirectional effects of children's externalizing behaviors on the quality of parenting the youth receive as perceived by their parents. Similarly, Stein and Polo, and Leung and Shek, each examine aspects of perceived parenting within the parent-child relationship and the impact of those perceptions on youth developmental and mental health outcomes in racially and culturally diverse families. In both cases, the researchers identified discrepancies between youth and their parents regarding perceptions of parenting and in each case these differences in perceptions were associated with negative emotional and behavioral sequelae in the youth. Even more intriguing is the fact that these comparable findings were generated from samples of persons in very different cultural contexts. Stein and colleagues' sample was comprised of Mexican and Mexican American families in the US while in the Leung and colleagues study the families were from Hong Kong.

In the next group of manuscripts, psychosocial factors impacting parents, youth and families are examined. Poverty and incarceration are two of the most difficult challenges explored within this context with researchers examining the impact of both on youth and families. Roberts, Snyder, Kaufman, Finley, Griffin, Anderson et al. report on the impact of parental arrest on children, specifying the ways in which children who experience a parental arrest suffer significant trauma and mental health problems. Similarly, Loper, Phillips, Nichols and Dalliare explore the nature of co-parenting relationships between incarcerated and non-incarcerated parents over time. Taylor, Budescu, 
Gebre and Hodzic illuminate the positive impact that social support from extended family can have toward reducing depressive symptoms in African American parents and youth facing significant financial burdens. Teen parenting, another "hot button" issue in popular culture is examined by Huang, Costeines, Ayala and Kaufman, among underresourced African American and Latina mothers via a study that seeks to determine risk and protective factors impacting their mental health and its relationship to their infant's development over time. Finally, Haskett, Neupert and Okado longitudinally examine parenting behavior stability and change in a sample of families where child protective services has become involved because of reports of child physical abuse.

One of the more clearly defined areas in the parenting literature is the correlational nature of parenting styles with behavioral and mental health outcomes for youth. LeCuyer examines this topic by looking at racial differences between African American and white parents related to limit setting for preschool aged children and surprisingly finds few racial differences. Voisin and Uji each examine the impact of parental behaviors and interpersonal styles on children's mental health. Voison, Hotton and Schneider find that parents who more frequently engage in verbal interpersonal aggression (with each other) have children who are more likely to be sexually active while Uji, Skamoto, Adaji and Kitamura find that adults who report being raised in authoritarian households as children experience worse mental health outcomes as adults. Gullan, LeRoy, Boxer and Mahoney find that familial engagement in positive parent child activities impacts child's mental health and that decreased positive activity engagement, coupled with a strong parent child alliance has negative impact on child internalizing problems. This finding is aligned with that of Laukkanen, Ojansuu, Tolvanen, Alatupa and Anuol who describe a relationship between child temperament (high positivity vs. low positivity) and the types of parenting employed by mothers (e.g., affection) in a sample of Finish families. As well, Buckholdt reports that parents who themselves struggle with emotion regulation and who respond inappropriately to their adolescent children, by discounting their emotions, set the stage for a host of teen emotional and behavioral problems. Finally, Lahlah, van der Knapp, Bogaerts and Lens assert the crosscultural relevance of long reported associations between perceived parenting style and patterns of juvenile delinquency in a sample of majority culture Dutch and Dutch Morroccan boys.

Physical health issues and mental health issues are known to impact parents and families whether they occur in the parents themselves or the youth. Moreira, Frontina, Bullinger and Canavarro highlight this in their manuscript focused on the impact of Type 1 childhood diabetes on the parent-child relationship. Similarly, Silva, Crespo and Canavarro examine the impact of pediatric asthma on the parent child relationship and parenting behaviors. Jee, Salter, Gonka and Chin also examine this concept on a more global level among Latino foster parents as she and her colleagues report on parental perceptions of caring for a child with a chronic health condition. Mental health concerns in parents and/or youth also have significant impact on the parent child relationship as evidenced by our contributors to this Special Issue. The authors focus on two of the most prevalent and impactful mental health concerns, depression and anxiety. Two research groups, McNeil, Harris-McKoy, Brantley, Fincham, Beach and McCullough and Shaffer each focus on the impact of maternal depression on children's negative acting out in racially and socioeconomically diverse families and Pereira, Barros, Mendonça, and Muris apply this interactional conceptualization to the impact of maternal anxiety on the development and expression of anxiety in children.

Each of the ways in which parenting is affected by psychosocial issues, health concerns and interpersonal styles might be addressed by interventions developed to help parents recognize, understand and improve their parenting skills. Haack, Gerdes and Lawton examine optimal mechanisms for increasing the likelihood that underserved Latino parents might engage in research on parent training. Murry, McNair, Myers, Chen and Brody build on this by examining the positive behavioral outcomes associated with a culturally targeted parenting intervention among rural low to middle income African American families. Similarly, Kimonis, Bagner, Linaris, Blake and Rodriguez examine outcomes of a parent training program on reductions in conduct problems among young children. The final article examines the effectiveness of a widely disseminated parent training program as implemented in a "real-world" setting. Specifically, Lanier, Kohl, Benz, Swinger, and Drake present findings from their research on a community based implementation of a widely used parent training approach, Parent Child Interaction Therapy (PCIT).

I hope that you find the information presented in this Special Issue helpful and that you gain new perspective on what parenting means for persons around the world at the beginning of the twenty first century.

\section{References}

Juang, L. P., Qin, D. B., \& Park, I. J. K. (2013). Deconstructing the myth of the "tiger mother": An introduction to the special issue on tiger parenting, Asian-heritage families, and child/adolescent well-being. Asian American Journal of Psychology, 4(1), 1-6. doi: $10.1037 / \mathrm{a} 0032136$.

Kim, S. Y., Wang, Y., Orozco-Lapray, D., Shen, Y., \& Murtuza, M. (2013). Does "tiger parenting" exist? Parenting profiles of 
Chinese Americans and adolescent developmental outcomes. Asian American Journal of Psychology, 4(1), 7-18. doi:10.1037/ a0030612.

Maccoby, E., \& Martin, J. (1983). Socialization in the context of the family: Parent-child interaction. In E. Hetherington (Ed.) (Series editor: P. H. Mussen), Handbook of child psychology (Formerly, Carmichael's manual of child psychology), Volume IV: Socialization, personality, and social development (4th ed., pp. 1-101). New York: Wiley. 